\title{
Optical Fiber Current Sensors in High Electric Field Environments
}

\author{
Allen H. Rose, Member, OSA, S. M. Etzel, and Kent B. Rochford, Member, IEEE, Member, OSA
}

\begin{abstract}
This paper analyzes the response of optical fiber current sensors that are subjected to high electric fields, such as fields encountered in gas-insulated systems. This paper shows that through the electrooptic (EO) Kerr effect, these fields can cause harmonic distortion of the measured ac current waveform. This harmonic distortion was confirmed experimentally. Also, this paper shows that it is possible to simultaneously measure both current and voltage waveforms and the phase between them using this effect. To minimize the electrooptic Kerr effect, optical fiber current sensors must be screened from high-electric fields.
\end{abstract}

Index Terms - Electric field effects, electrooptic Kerr effect, gas insulated systems, optical fiber current sensor, polarimetric, Sagnac.

\section{INTRODUCTION}

$\mathbf{T}$ HE response of optical current sensors placed in high electric field environments, such as gas-insulated systems (GIS), can be influenced by the electrooptic (EO) Kerr effect. The EO Kerr effect is an electric-field-induced linear birefringence that arises when an electric field changes the polarizability of the glass molecules [1]. Linear birefringence, either from stress, bending, waveguide form, or the EO Kerr effect, alters the response of a Faraday effect current sensor (a sensor based on a magnetic-field-induced circular birefringence) [2]. In this paper, we analyze the EO Kerr effect on polarimetric and interferometric Sagnac optical fiber current sensors in high electric fields. We also show experimentally the harmonic distortion, induced by the EO Kerr effect, on a polarimetric fiber current sensor.

Optical fiber current sensors have several economic and performance advantages over conventional current transducers in fault detection and metering. The bandwidth and high dynamic range of these sensors provide the power utility engineer with a diagnostic tool for evaluating generators and the transmission grid [3], [4]. Due to their all-dielectric design, installation costs are significantly lower, and sensor failure does not pose a threat to power utility personnel or equipment. Only recently, after almost three decades of development, are these sensors entering the market [3]-[10]. The GIS environment or other highelectric potential applications are where optical sensors have the greatest economic advantage, but no study of the EO Kerr effect on these sensors has been made.

Manuscript received January 18, 1998; revised March 17, 1999.

The authors are with the Optoelectronics Division, the National Institute of Standards and Technology (NIST), Boulder, CO 80303 USA.

Publisher Item Identifier S 0733-8724(99)04505-3.
Over the last two decades, there have been field tests of optical current sensors in GIS environments [11]-[16]. However, the EO Kerr effect on these sensors was not noted or detected. Some reasons for not detecting the EO Kerr effect in these current sensors are low or nonuniform electric fields, short optical path, and polarizer alignment.

The EO Kerr effect has been used for many years to measure high voltages and electric fields [17], [18]. Most applications are with liquids that have a large temperature dependence, due to the nonlinear polarizability of the molecules in the liquid or glass, and require the proximity of electrodes [18], [19]. In fibers the EO Kerr effect has been used for voltage sensing and modulating the polarization state [20], [21]. However, the temperature dependence of the EO Kerr effect in fiber and the difficulty of making a stable sensor with low stress sensitivity have discouraged its use [21].

In this paper, we also show that a conventional polarimetric fiber current sensor, placed in a high electric field, can be used to simultaneously measure the current and voltage waveforms and the phase between them. This is accomplished by the proper arrangement of the polarizer/analyzer angles and separating the harmonics produced by the applied current and voltage. In power transmission and distribution, current and voltage are typically measured with separate transducer heads, both with conventional electrically coupled sensors and optical sensors [4], [11], [12], [7]-[9], [22], [23]. The simultaneous measurement of voltage and current, using the EO Kerr effect or Pockels effect, has been previously shown [24]-[26]. However, none of these sensor designs has been demonstrated in a high power utility application. Although we do not demonstrate a fiber sensor using the EO Kerr effect in a high power utility application, we simulate the high power environment in the laboratory. Also, our sensor arrangement does not require two detection systems [26], a magnetostrictive element [24], or a modified Sagnac interferometer with bulk elements [25].

\section{THEORY}

\section{A. Polarimetric Sensors}

The output polarization state of a fiber current sensor with linear birefringence, a linear input polarization state at $\theta$, and an output polarizer at $\beta$ is found using Jones' calculus as shown in (1) at the bottom of the next page [16], [27]. In (1), $\delta$ is the retardance due to linear birefringence, $\rho$ is the circular retardance due to the Faraday effect, and $\Delta=\left(\delta^{2}+4 \rho^{2}\right)^{\frac{1}{2}}$ [16]. We will assume $\delta=\delta_{s}+\delta_{\mathrm{EOK}}$ where $\delta_{s}$ is the static 
retardance, $\delta_{\mathrm{EOK}}$ is the $\mathrm{EO}$ Kerr retardance, and the retardance axes are at $0^{\circ}$. A fiber current sensor with multiple turns around a conductor has $\rho$ equal to $\mu V N I$ (where $\mu V$ is the Verdet constant in units of $\mathrm{rad} / \mathrm{A}, N$ the number of fiber turns around the conductor, and $I$ the electric current [8]). The output intensity of this system, $I_{o}=\left|E_{\mathrm{ox}}\right|^{2}+\left|E_{\mathrm{oy}}\right|^{2}$, is [28], [29]

$$
\begin{aligned}
I_{o}=\frac{1}{2} & +\rho \frac{\sin \Delta}{\Delta} \sin (2 \beta-2 \theta) \\
& +\frac{\delta^{2}}{8} \frac{\sin ^{2}(\Delta / 2)}{(\Delta / 2)^{2}} \cos (2 \beta+2 \theta) \\
& +\frac{1}{2}\left(\frac{\delta^{2}}{\Delta^{2}} \cos ^{2}(\Delta / 2)+\frac{4 \rho^{2}}{\Delta^{2}} \cos \Delta\right) \cos (2 \beta-2 \theta) .
\end{aligned}
$$

Typically, a current sensor has $\beta$ and $\theta$ set so that $\sin (2 \beta-$ $2 \theta)= \pm 1$ when $\pm \beta= \pm \pi / 4+\theta$ and $\cos (2 \beta-2 \theta)=0$. Also, the input polarization axis is aligned with the retardance axis $\left(\theta=0^{\circ}\right)$, so $\cos (2 \beta+2 \theta)=0$. Thus, with the typical arrangement an optical current sensor will have a "differenceover-sum" response of $2 \rho \sin (\Delta) / \Delta$ [16]. (The difference $I_{\Delta}$ is $I_{o}(+\beta)-I_{o}(-\beta)$. The sum $I_{\Sigma}$ is $I_{o}(+\beta)+I_{o}(-\beta)$.) If $\theta \neq 0$ and $\pm \beta= \pm \pi / 4+\theta$ then the "difference-over-sum" response of the sensor can be expressed as

$$
\frac{I_{\Delta}}{I_{\Sigma}}=2 \rho \frac{\sin (\Delta)}{\Delta}+\frac{\delta^{2}}{4} \frac{\sin ^{2}(\Delta / 2)}{(\Delta / 2)^{2}} \cos (2 \beta+2 \theta) .
$$

Also, if $\sin (2 \beta-2 \theta)=1$ is held while $\theta$ is rotated, a value of $\theta=\theta^{\prime}$ can be found such that $\cos \left(2 \beta+2 \theta^{\prime}\right)=1$ [28]. At $\theta^{\prime}$ the response of the current sensor has a maximum sensitivity to changes in $\delta$, via temperature, vibration, and the EO Kerr effect.

The retardance due to the EO Kerr effect has the functional form $\delta_{\mathrm{EOK}}=2 \pi L K E^{2}$, where $L$ is the length of the optical path in the field, $K$ is the EO Kerr constant equal to $(5.3 \pm 0.2) \times 10^{-16} \mathrm{~m} / \mathrm{V}^{2}$ at $23^{\circ} \mathrm{C}$ in silica, and $E$ is the electric field strength [21], [30]. $\delta_{\mathrm{EOK}}$ becomes large enough to affect the response of an optical fiber current sensor when $L \approx 10 \mathrm{~m}$ and when the sensor is placed where $E_{o} \geq 1$ $\mathrm{MV} / \mathrm{m}$ rms, such as the GIS environment. For exposed or air-cooled power lines the electric field typically is less than $\sim 120 \mathrm{kV} / \mathrm{m} \mathrm{rms}$. Under these conditions the effects of $\delta_{\mathrm{EOK}}$, for fiber lengths from 1 to $10 \mathrm{~m}$, are below the noise floor of the current sensor and not measurable. Because $K$ has a large temperature dependence [21], $\delta_{\mathrm{EOK}}$ increases the temperature dependence of an optical sensor.

When an optical fiber current sensor is placed in a high electric field and the polarizers are fixed so that (3) applies, the response of the sensor will have additional harmonics of the fundamental frequency $\omega$. For $I=I_{o} \sin (\omega t)$ and
$E=E_{o} \sin (\omega t+\phi)$, (3) can have the form, $I_{\Delta} / I_{\Sigma} \approx$ $A \sin (\omega t)+B \sin (\omega t+2 \phi)+C \sin (2 \omega t+2 \phi)+D \sin (3 \omega t+$ $2 \phi)+E \sin (4 \omega t+4 \phi)$ where

$$
\begin{aligned}
& A=2 \rho_{o}-\frac{\delta_{s}^{2} \rho_{o}}{60}-\frac{3 \delta_{\mathrm{EOK}}^{2} \rho_{o}}{480}-\frac{\delta_{s} \delta_{\mathrm{EOK}} \rho_{o}}{60} \\
& B=-D=-\frac{\delta_{\mathrm{EOK}} \rho_{o}\left(2 \delta_{s}+\delta_{\mathrm{EOK}}\right)}{240} \\
& C=\frac{\delta_{\mathrm{EOK}} \delta_{s}}{2} \cos (2 \beta+2 \theta) \\
& E=\frac{\delta_{\mathrm{EOK}}^{2}}{8} \cos (2 \beta+2 \theta)
\end{aligned}
$$

$\phi$ is the phase between the current and voltage, $\rho_{o}$ is $\mu V N I_{O}$, and we assume $\delta_{s}<1, \delta_{\mathrm{EOK}}<1$. Minor components of the power series expansion of (3), powers of $\delta_{s}, \delta_{\mathrm{EOK}}$, and $\rho$ larger than two, and higher frequencies are neglected. The odd-harmonic components, $A, B$, and $D$, arise from the first term in (3) and the even-harmonic components, $C$ and $E$, arise from the second term in (3). $C$ and $E$ depend on the polarizer alignment relative to the axis of $\delta_{s}$ and can be made zero. $B$, $C$, and $D$ depend directly on $\delta_{s}$. Even with $\delta_{s}=0$ the EO Kerr effect will have a signal at the third and fourth harmonics.

For example, a fiber sensor, with $N=8$ and operating at $830 \mathrm{~nm}$ [11], placed in a GIS with $550 \mathrm{kV} \mathrm{rms}, 4.5$ $\mathrm{kA}$ rms, operating at $60 \mathrm{~Hz}$, would have a $0.5-\mathrm{m}$ diameter outer conductor [31] so that $L=12.7 \mathrm{~m}$. At $830 \mathrm{~nm}$, $\mu V=2.58 \mu \mathrm{rad} / \mathrm{A}$ [32], so that at full current $\rho_{O}$ is $93 \mathrm{mrad}$. With $E=3.3 \mathrm{MV} / \mathrm{m} \mathrm{rms}$, at full voltage, $\delta_{\mathrm{EOK}}$ is $460 \mathrm{mrad}$. Assuming $\delta_{s}=174 \mathrm{mrad}\left(10^{\circ}\right)$ and with the above values, $(A+B)$ is about $184 \mathrm{mrad} \mathrm{rms}, C$ is about $40 \mathrm{mrad} \mathrm{rms}, D$ is about $142 \mu \mathrm{rad} \mathrm{rms}$, and $E$ is about $26 \mathrm{mrad} \mathrm{rms}$. For this example, the ratio $\delta_{\mathrm{EOK}} / \rho$ is about 5 .

Fig. 1 shows (3), with the values above, for cases where $\cos \left(2 \beta+2 \theta^{\prime}\right)=1, \cos (2 \beta+2 \theta)=0$, and the voltage is off with $\delta_{s}=0$. In all cases $\phi=0$. The optical response in Fig. 1 shows significant distortion due to the second and fourth harmonic terms $(C$ and $E$ ). For the magnitude of these harmonics to be insignificant (less than $0.1 \%$ of the fundamental), $\delta_{\mathrm{EOK}}$ would need to be less than $2 \mathrm{mrad}$, or $\delta_{\mathrm{EOK}} / \rho<0.02$, or $E$ would need to be less than 220 $\mathrm{kV} / \mathrm{m}$ rms. Even when the sensor polarizers are aligned so that the second term in (3) is 0 , the response waveform is distorted by the electric field, through $\delta_{\mathrm{EOK}}$ (terms $A, B$, and $D$ ) as shown by the dashed line in Fig. 1. Thus, when $E$ is large enough $\delta_{\mathrm{EOK}}$ will effect a current measurement in an optical fiber sensor regardless of polarizer arrangement. Polarizer arrangements where $\theta \neq 0$ will only increase the effect of $\delta_{\mathrm{EOK}}$ on the sensor response.

$C$ and $E$ could be used to measure the applied voltage and phase when the applied current does not have harmonic

$$
\left(\begin{array}{l}
E_{\mathrm{ox}} \\
E_{\mathrm{oy}}
\end{array}\right)=\left(\begin{array}{cc}
\cos ^{2}(\beta) & \frac{\sin (2 \beta)}{2} \\
\frac{\sin (2 \beta)}{2} & \sin ^{2}(\beta)
\end{array}\right) \times\left(\begin{array}{cc}
\cos (\Delta / 2)+i \frac{\delta}{2} \frac{\sin (\Delta / 2)}{(\Delta / 2)} & -\rho \frac{\sin (\Delta / 2)}{(\Delta / 2)} \\
\frac{\rho \sin (\Delta / 2)}{(\Delta / 2)} & \cos (\Delta / 2)-i \frac{\delta}{2} \frac{\sin (\Delta / 2)}{(\Delta / 2)}
\end{array}\right) \times\left(\begin{array}{c}
\cos (\theta) \\
\sin (\theta)
\end{array}\right)
$$




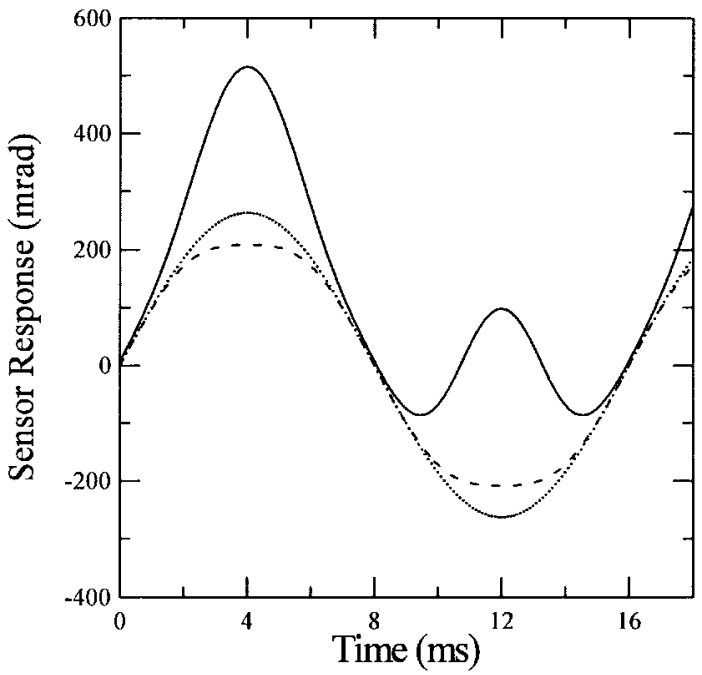

Fig. 1. The calculated response of a polarimetric optical fiber sensor in a $550 \mathrm{kV} \mathrm{rms}, 4.5 \mathrm{kA} \mathrm{rms}, 60 \mathrm{~Hz}$ GIS with $\delta_{\mathrm{EOK}} / \rho=5$. The solid line has $\cos \left(2 \beta+2 \theta^{\prime}\right)=1$, dashed line has $\cos (2 \beta+2 \theta)=0$, and dotted line has $\delta_{\mathrm{EOK}}=\delta_{s}=0$.

content at $2 \omega$ and $4 \omega$ that would interfere with the measurement. However, a voltage measurement using $C$ or $E$ will be further complicated by the temperature dependence


and $\delta_{s},\left(1 / \delta_{s}\right)\left(d \delta_{s} / d T\right)= \pm(5.7 \pm 0.2) \times 10^{-4} /{ }^{\circ} \mathrm{C}[33]$ The temperature dependences of $A+B, C, D$, and $E$ are

$$
\begin{aligned}
\frac{1}{(A+B)} \frac{d(A+B)}{d t} \approx & \frac{1}{V} \frac{d V}{d T}-\frac{\delta_{s}}{3} \frac{d \delta_{s}}{d T} \\
& -\frac{\delta_{\mathrm{EOK}}^{2}}{3}\left(\alpha+\frac{1}{K} \frac{d K}{d T}\right) \\
& \approx\left(69-190 \delta_{s}^{2}-1866 \delta_{\mathrm{EOK}}^{2}\right) \\
& \times 10^{-6 /{ }^{\circ} \mathrm{C}} \\
\frac{1}{C} \frac{d C}{d T} & =\frac{1}{d \delta_{s}} \frac{d \delta_{s}}{d T}+\alpha+\frac{1}{K} \frac{d k}{d T} \\
& \approx 0.6 \% /{ }^{\circ} \mathrm{C} \\
\frac{1}{D} \frac{d D}{d T} & =\frac{1}{V} \frac{d V}{d T}+\frac{1}{d \delta_{s}} \frac{d \delta_{s}}{d T}+\alpha+\frac{1}{k} \frac{d k}{d T} \\
& \approx 0.62 \% /{ }^{\circ} \mathrm{C} \\
\frac{1}{E} \frac{d E}{d T} & =2 \alpha+\frac{2}{K} \frac{d K}{d T} \\
& \approx 1.12 \% /{ }^{\circ} \mathrm{C} .
\end{aligned}
$$

We have assumed that $\delta_{s}<1, \delta_{\mathrm{EOK}}<1, \rho<\delta, \phi=0$, and that the temperature dependence of $\delta_{s}$ is positive. Also, $\alpha$ is the thermal expansion coefficient of silica glass $\left(0.55 \times 10^{-6} /{ }^{\circ} \mathrm{C}\right.$ [34]) and the temperature dependence of the Verdet constant is $69 \times 10^{-6 /{ }^{\circ} \mathrm{C}}$ [35]. The temperature dependence of $K$ dominates the second through fourth harmonic signals' temperature dependence. To be useful, these signals would need temperature correction. The temperature dependence of $\delta_{\mathrm{EOK}}$ could reduce or compensate for the temperature dependence of $\mu V$ in a current measurement when $(192 \mathrm{mrad}$ $\left.>\delta_{\mathrm{EOK}}>30 \mathrm{mrad}\right)$ or $\left(69 \times 10^{-6 /{ }^{\circ} \mathrm{C}}>1866 \delta_{\mathrm{EOK}}^{2} \times\right.$

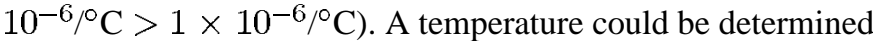
from $C$ and $E$, to correct a voltage measurement. Filtering the ac and dc components of (3) has been used to compensate the temperature dependence of $\delta_{s}$ in these fiber current sensors [28].

The phase $\phi$ is equivalent to the phase between $\omega$ and $2 \omega$ components, and can be measured by performing a Hilbert transform on the response waveform [36]. Selection of individual harmonic signals after a fast Fourier transform (FFT) and followed by an inverse FFT are performed to create an analytic form for each frequency. The phase difference between the applied current and voltage is determined from the analytic form. Depending on the value of $\delta_{s}$, either $C$ or $E$ can be used with $(A+B)$ to measure the voltage, and with the current and phase values an estimate of the electric power can be made.

Our example sensor in a GIS environment, with a noise equivalent signal of $1 \mu \mathrm{rad} / \sqrt{\mathrm{Hz}}$, would have a noise equivalent electric power (NEP) of $\sim 66 \mathrm{~W}$ with a $1 \mathrm{~Hz}$ bandwidth, when $C$ is used to measure the voltage. If $E$ was used to measure the voltage, the NEP would be about $1 \mathrm{~kW}$. (The noise equivalent voltage is different for $C$ and $E$ because of their nonlinear response.) The average power is about $2.5 \mathrm{GW}$, when $\phi=0$, for a $550 \mathrm{kV}, 4.5 \mathrm{kA}$ GIS line.

\section{B. Interferometric Sensors}

A loop Sagnac current sensor will not have the same response or dependence on retardance as a polarimetric sensor would have, but will still have a distorted waveform due to $\delta_{\mathrm{EOK}}$. The response function of a loop Sagnac current sensor is [37], [38]

$$
\Delta \phi_{\text {loop }}=2 \rho \frac{\tan (\Delta)}{\Delta}
$$

where $\Delta \Phi_{\text {loop }}$ loop is the measured phase shift in the interferometer. An in-line Sagnac current sensor has a similar response except that $\Delta \phi_{\text {in-line }}=2 \Delta \phi_{\text {loop }}$ [37]. Fig. 2 shows the response of the loop Sagnac current sensor using (6) with the previous values used for the polarimetric sensor in a high electric field environment. The two cases presented in the figure have the voltage off with $\delta_{s}=0$ and the voltage on with $\delta_{s}=174 \mathrm{mrad}\left(10^{\circ}\right)$.

Equation (4) can be written so that $\Delta \phi \approx A_{S} \sin (\omega t)+$ $B_{S} \sin (\omega t+2 \phi)+C_{S} \sin (3 \omega t+2 \phi)$, where

$$
\begin{aligned}
& A_{S}=2 \rho_{o}+\frac{\delta_{s}^{2} \rho_{o}}{60}+\frac{3 \delta_{\mathrm{EOK}}^{2} \rho_{o}}{480}+\frac{\delta_{s} \delta_{\mathrm{EOK}} \rho_{o}}{60} \\
& B_{S}=C_{s}=\frac{\delta_{\mathrm{EOK}} \rho_{o}\left(2 \delta_{s}+\delta_{\mathrm{EOK}}\right)}{240}
\end{aligned}
$$

assuming $\delta_{s}<1$ and $\delta_{\mathrm{EOK}}<1$. Minor components of the power series expansion of (6), powers of $\delta_{s}, \delta_{\mathrm{EOK}}$, or $\rho$ greater than 2, and higher frequencies are neglected. For the terms in $A_{S}$ and $B_{S}$, terms that depend on $\delta_{\mathrm{EOK}}$, to distort the response of the Sagnac sensor by less than $0.1 \%, \delta_{\mathrm{EOK}}$ must be less than $276 \mathrm{mrad}$, when $\delta_{s}=174 \mathrm{mrad}\left(10^{\circ}\right)$ and $\phi=0$. For our example sensor, $E$ would need to be less than $2.7 \mathrm{MV} / \mathrm{m}$ rms. For $C_{S}$ to effect the sensor's response by less than $0.1 \%$, $\delta_{\mathrm{EOK}}$ must be less than $690 \mathrm{mrad}$. 


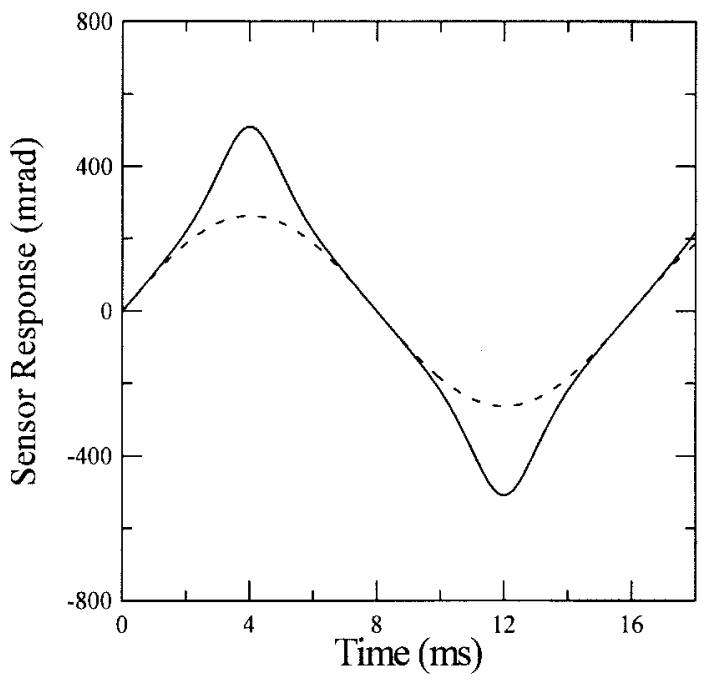

Fig. 2. The calculated response of a loop Sagnac current sensor in a 550 $\mathrm{kV}$ rms, $4.5 \mathrm{kA} \mathrm{rms}, 60 \mathrm{~Hz}$ GIS. The solid line has the voltage on and the dashed line shows $\delta_{\mathrm{EOK}}=\delta_{s}=0$.

The temperature dependence of $\left(A_{S}+B_{S}\right)$ is similar to the temperature dependence of $(A+B)$ and is

$$
\begin{aligned}
& \frac{1}{\left(A_{S}+B_{S}\right)} \frac{d\left(A_{S}+B_{S}\right)}{d T} \\
& \approx \frac{1}{V} \frac{d V}{d T}+\frac{2 \delta_{s}}{3} \frac{d \delta_{s}}{d T}+\frac{2 \delta_{\mathrm{EOK}}^{2}}{3}\left(\alpha+\frac{1}{K} \frac{d K}{d T}\right) \\
& \approx\left(69+380 \delta_{s}^{2}+3733 \delta_{\mathrm{EOK}}^{2}\right) \times 10^{-6} /{ }^{\circ} \mathrm{C} .
\end{aligned}
$$

We have assumed that $\delta_{s}<1, \delta_{\mathrm{EOK}}<1, \rho<\delta$, and that the temperature dependence of $\delta_{s}$ is positive. For the $\delta_{\mathrm{EOK}}$ term to be less than $1 \times 10^{-6}, \delta_{\mathrm{EOK}}$ should be less than $16 \mathrm{mrad}$. A Sagnac current sensor's temperature dependence increases rapidly as $\delta_{\mathrm{EOK}}$ increases beyond $16 \mathrm{mrad} . C_{S}$ has the same temperature dependence as $D$.

\section{Simulated GIS ENVIRONMENT}

We have designed an apparatus to safely simulate the GIS environment in the laboratory using low power electric circuits. A schematic of the circuit is shown in Fig. 3(a) along with a sketch of the optical fiber current sensor placement. The branches of the circuit are inductively and capacitively coupled, so that low power is flowing in each. One branch provides about $100 \mathrm{~A}$ rms with a potential less than $1 \mathrm{~V}$ rms. The other branch provides about $2.5 \mathrm{kV}$ rms with a current less than $1 \mathrm{~mA} \mathrm{rms}$. The circuits were operated at a frequency of $100 \mathrm{~Hz}$. The annealed optical fiber is placed into the gap formed by the disc and ring, as shown in Fig. 3(b). The gap is about $2.5 \mathrm{~mm}$ wide and filled with a silicone dielectric gel. Fields were limited to about $1 \mathrm{MV} / \mathrm{m}$ rms between the disc and ring because of arcing across the top surface of the gel. Even though this field strength is below the lowest GIS field of about $1.27 \mathrm{MV} / \mathrm{m}$ rms [31], we were able to demonstrate EO Kerr effect in an optical fiber current sensor. (a)

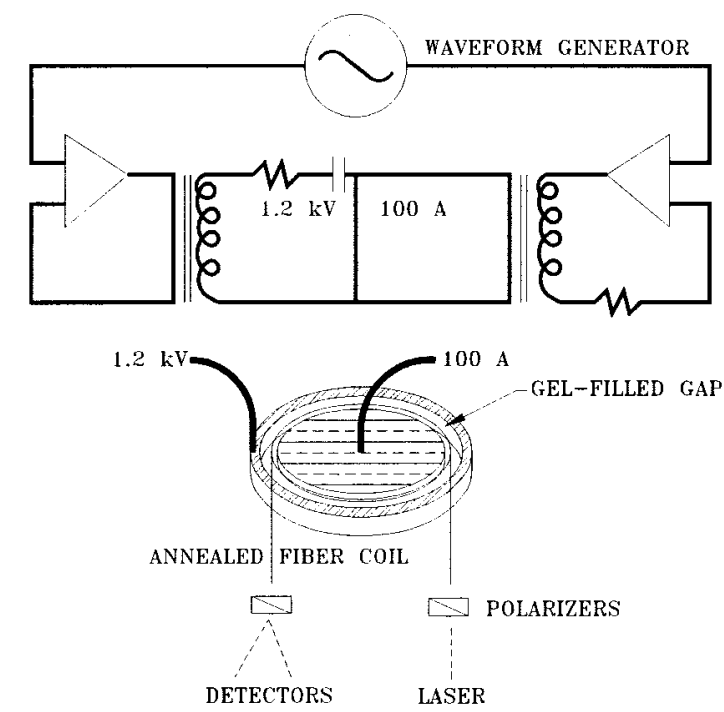

Fig. 3. (a) A schematic of the circuit used to simulate GIS conjditions. (b) A diagram showing the placement of the annealed fiber coil and electric power.

\section{EXPERIMENTAL RESULTS}

An optical fiber coil with $N \approx 26$ and $\sim 15 \mathrm{~cm}$ diameter was twisted and annealed to reduce the linear retardance $\delta_{s}$ to about $471 \mathrm{mrad}\left(27^{\circ}\right)$ [2], [39]. The value of $\delta_{s}$ was confirmed with the measured and predicted magnitude of $2 \rho \sin \delta_{s} / \delta_{s}$ and by the rotating polarizer method [40]. Also, the birefringence axes of $\delta_{\mathrm{EOK}}$ and $\delta_{s}$ coincide within $\pm 1^{\circ}$. The length $L$ of the fiber in the field is about $11.6 \mathrm{~m}$. (Some of the fiber turns did not lie in the gap between the disc and ring, shortening the field interaction length.) The single-mode fiber was operated at a wavelength of $636 \mathrm{~nm}$ so that values of $\rho$ similar to values found in GIS applications could be generated. The input polarizer was set so $\cos \left(2 \beta+2 \theta^{\prime}\right) \approx 1$, and the EO Kerr signals remained at a maximum.

With $\sim 120 \mathrm{~A}$ rms passing through the disc, $\rho$ is about $14 \mathrm{mrad}$. (The Verdet constant $\mu V$ at $636 \mathrm{~nm}$ is $4.58 \pm$ $0.01 \mu \mathrm{rad} / \mathrm{A}$ [32].) With $\sim 2.48 \mathrm{kV}$ rms applied to the ring $(E \approx 992 \mathrm{kV} / \mathrm{m} \mathrm{rms}) \delta_{\mathrm{EOK}}$ is about $38 \mathrm{mrad}$. These values of $\rho$ and $\delta_{\mathrm{EOK}}$ give a ratio $\delta_{\mathrm{EOK}} / \rho$ of about 3 . This ratio $\delta_{\mathrm{EOK}} / \rho$ is near that of a sensor, similar to the one described in the theory section, in a $300 \mathrm{kV}, 3 \mathrm{kA}$, GIS environment. By reducing the current or $\rho$, we could increase the ratio to simulate other GIS environments, but in this case our $\delta_{\mathrm{EOK}}$ was about a factor of 10 smaller than a sensor placed in typical GIS applications.

The response of our sensor taken with a signal analyzer is shown in Fig. 4. Three harmonics of the fundamental drive frequency, at $100 \mathrm{~Hz}$, are many decibels above the noise floor. The $*$ marks on each peak show predicted values from $A+B$, $C, D$, and $E$. The predicted and measured signals at $300 \mathrm{~Hz}$ do not agree in large part because our current source has a third-harmonic component. Both applied current and voltage can be measured, from the magnitude of the 100, 200, or 400 $\mathrm{Hz}$ signals, if the current does not have significant harmonic (greater than $0.1 \%$ ) content.

From the $100 \mathrm{~Hz}$ signal in Fig. 4 a noise equivalent current of about $2.6 \mathrm{~mA} / \sqrt{\mathrm{Hz}}$ can be determined. From the $200 \mathrm{~Hz}$ 


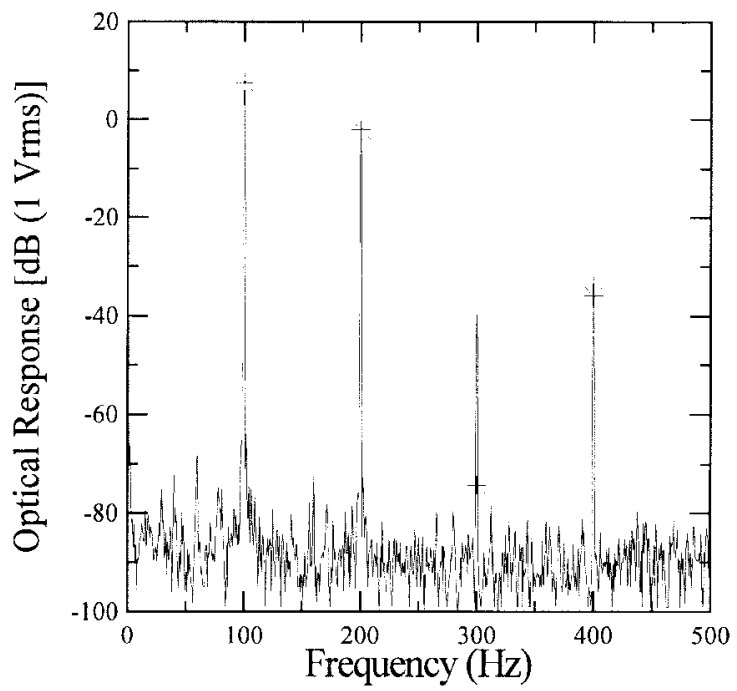

Fig. 4. The spectral response of our sensor with $\sim 992 \mathrm{kV} / \mathrm{m} \mathrm{rms}$ and $\sim 120$ A rms at $100 \mathrm{~Hz} . \delta_{\mathrm{EOK}} / \rho \approx 3$ and the "**" marks show the predicted values.

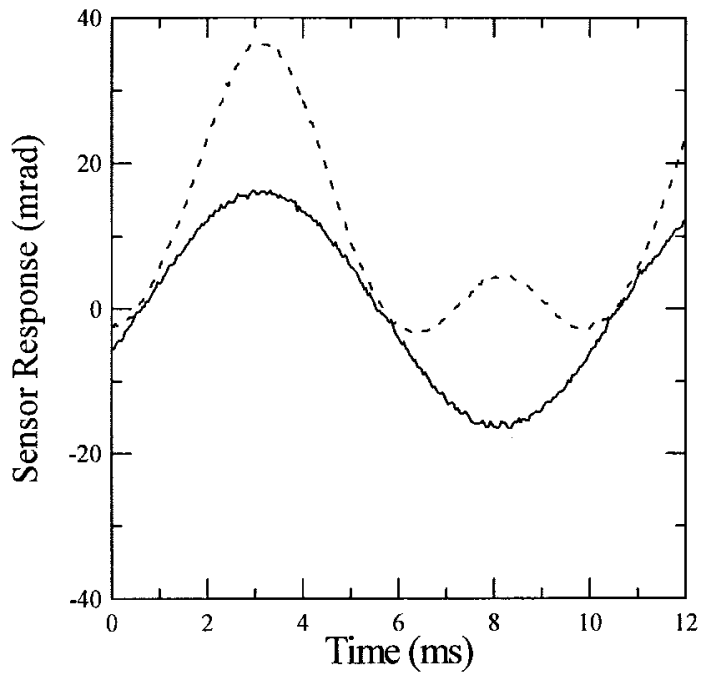

Fig. 5. Response of our sensor with $\sim 900 \mathrm{kV} / \mathrm{m} \mathrm{rms}$ and $\sim 50 \mathrm{~A}$ rms at 100 $\mathrm{Hz}$, or with $\delta_{\mathrm{EOK}} / \rho \approx 5$. The dashed line is th sensor with $\cos \left(2 \beta+2 \theta^{\prime}\right) \approx 1$ and the voltage is on. The solid line is with the voltage off.

signal, a noise equivalent voltage (NEV) of about $15 \mathrm{~V}$ with a 1 $\mathrm{Hz}$ bandwidth can be determined. The $400 \mathrm{~Hz}$ signal provides an NEV of approximately $420 \mathrm{~V}$. The difference in the two NEV's is due to the nonlinear nature of the 200 and $400 \mathrm{~Hz}$ response functions. From these noise equivalent values a NEP can be estimated for our sensor at about $40 \mathrm{~mW}$ when using the $200 \mathrm{~Hz}$ voltage signal, and about $1 \mathrm{~W}$ when using the $400 \mathrm{~Hz}$ voltage signal. The apparent measured average electric power at the sensor is about $298 \mathrm{~kW}$ rms, when $\phi=0$. Our noise equivalent $\rho$ was less than $1 \mu \mathrm{rad} / \sqrt{\mathrm{Hz}}$ for the current signal shown in Fig. 4.

Fig. 5 shows the wave form of this sensor with the current reduced to about $50 \mathrm{~A} \mathrm{rms}$ and the voltage at about $2.25 \mathrm{kV}$ or $E \approx 900 \mathrm{kV} / \mathrm{m} \mathrm{rms}$. This gives a $\delta_{\mathrm{EOK}} / \rho$ ratio of about five, similar to Fig. 1. The phase between the voltage and current is about $91^{\circ}$. In the figure the solid line corresponds to the voltage off and the dashed line corresponds to the voltage on. The addition of a second harmonic is apparent in the asymmetric waveform and similar to the shape of the

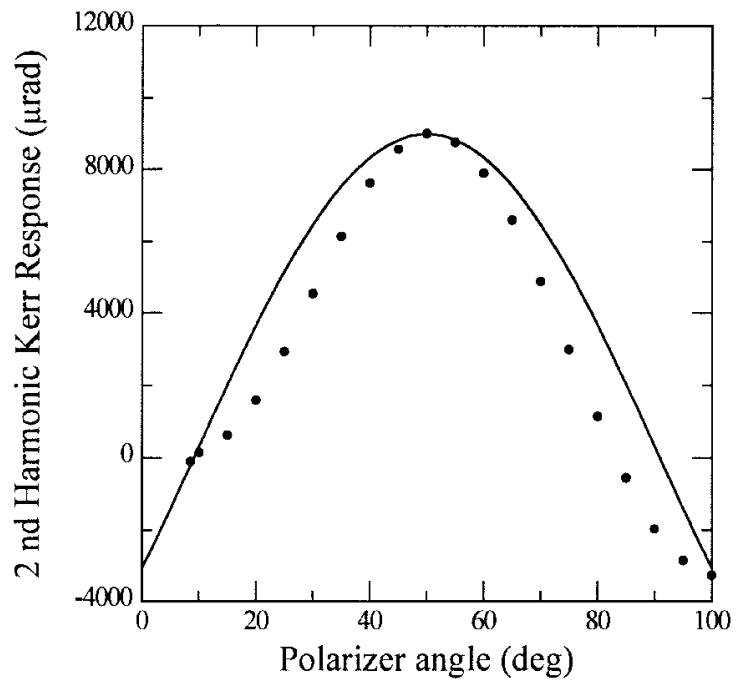

Fig. 6. The input polarization angle dependence of the second-harmonic EO Kerr signal. The solid line is a normalized $\sin (2 \theta)$ function.



Fig. 7. The field dependence of our sensor's second-harmonic signal. The solid line is the predicted value with $\delta_{s} \approx 27^{\circ}, L \approx 11.6 \mathrm{~m}$, and $\cos \left(2 \beta+2 \theta^{\prime}\right) \approx 1$.

theoretical waveform shown in Fig 1 . In Fig. $1, \delta_{\mathrm{EOK}}$ is 460 $\mathrm{mrad}$, which is large enough to distort the waveform of a sensor even with the polarizers aligned so that $\cos (2 \beta+2 \theta)=$ 0 . In our sensor $\delta_{\mathrm{EOK}} \approx 38 \mathrm{mrad}$, too small for $\delta_{\mathrm{EOK}}$ to visibly distort the measured waveform when the polarizers are so aligned.

To measure the phase $\phi$ between the applied current and voltage we recorded the optical waveform and separately measured $\phi$ electrically. Then we performed a Hilbert transform to separate the fundamental and the second harmonic so that the magnitude and phase of each could be determined. The uncertainty of the optical method of determining the phase was $\pm 0.2^{\circ}$. The optical and electrical phase measurements differed by about $6^{\circ}$ because of additional phase in the electrical probes.

Fig. 6 shows the angle dependence of the sensor's secondharmonic signal as the input polarizer is rotated. A normalized $\sin (2 \theta)$ function is shown for comparison. For each mea- 


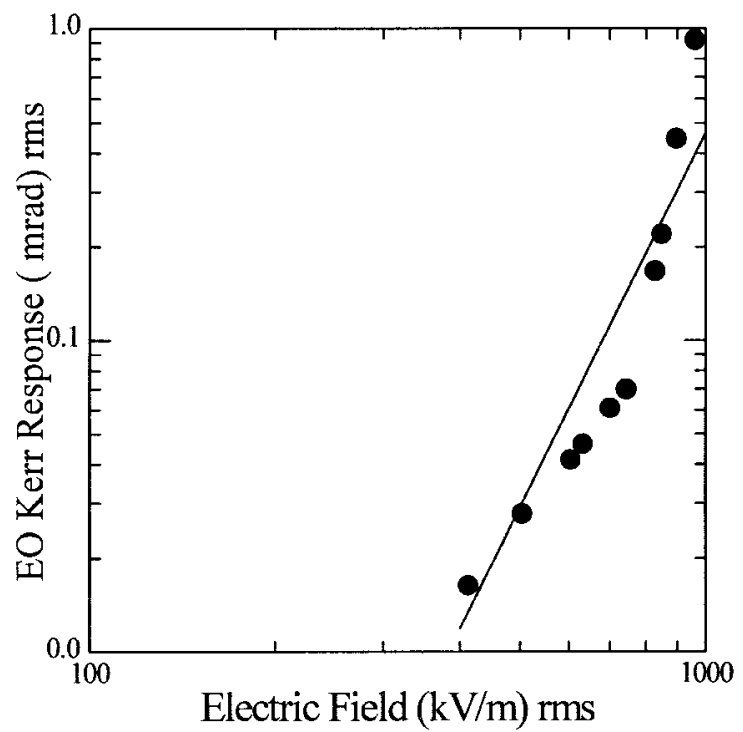

Fig. 8. The field dependence of our sensor's fourth-harmonic signal. The solid line is the predicted value with $L \approx 11.6 \mathrm{~m}$, and $\cos \left(2 \beta+2 \theta^{\prime}\right) \approx 1$.

surement the beam-splitting analyzer is rotated so that the dc difference between the two photodetectors is near 0 , or $\cos (2 \beta-2 \theta)=0$. Also, for each measurement the signal is normalized by the total optical transmittance $I_{\Sigma}$.

The voltage dependence of the second and fourth harmonic signals is shown in Figs. 7 and 8. The predicted values of $C$ and $E$ are plotted using the values of $\delta_{s}, L$, and $E$ given above, and the polarizers are arranged so that the EO Kerr signal is maximized. The data are in measurable agreement with the theoretical predictions. A least-squares fit to the data gives a power dependence of $E^{n}$ with $n=1.97$ for the second harmonic and $n=3.86$ for the fourth harmonic. These values agree with the theoretical values of two and four within the least-squares fit uncertainty.

\section{CONCLUSION}

As optical fiber sensors are used more in power industry GIS applications, the EO Kerr effect will need to be considered in the design and installation of both polarimetric and interferometric sensors. Current sensors placed in high electric fields or GIS environments will have either a response distortion or altered temperature dependence due to the retardance produced by the EO Kerr effect. For minimum EO Kerr effects, polarizer alignment and sensor placement must be considered. A metering class optical fiber current sensor on a high voltage line will need a screened low electric field location to minimize this effect. If the sensor is placed in a GIS with a high electric field and no screening, it should be located where the temperature is stable. This distortion of the ac current waveform can easily become significant, greater than $0.1 \%$, with only a few turns of fiber or lengths approaching $10 \mathrm{~m}$. For the waveform distortion to be greater than $0.1 \%$ in the Sagnac or typically aligned polarimetric sensor, electric fields $>2.7 \mathrm{MV} / \mathrm{m}$ are required when $L \approx 12 \mathrm{~m}$.

A polarimetric sensor, with polarizers aligned to maximize the harmonics signals generated by the EO Kerr effect, can be used to simultaneously monitor the current, voltage, and phase of a GIS power line. However, the temperature dependence of these harmonics is near $1 \% /{ }^{\circ} \mathrm{C}$ and harmonics on the ac current waveform can make these measurements difficult.

In high electric field environments, the Sagnac current sensor may have a higher stability and less EO Kerr waveform distortion than the polarimetric sensor. However, the polarimetric sensor has a smaller temperature dependence and can even have some temperature compensation of the Verdet constant temperature dependence. The Sagnac's temperature dependence, $1 /\left(A_{S}+B_{S}\right) * d\left(A_{S}+B_{S}\right) / d T$, will increase more than $1 \times 10^{-6} /{ }^{\circ} \mathrm{C}$ with fields as small as $600 \mathrm{kV} / \mathrm{m}$. The polarimetric current sensor's temperature dependence, $1 /(A+$ $B) * d(A+B) / d T$, will not increase more than $1 \times 10^{-6} /{ }^{\circ} \mathrm{C}$ until fields $>2.5 \mathrm{MV} / \mathrm{m}$ are reached for polarizers aligned to minimize the EO Kerr effect.

\section{ACKNOWLEDGMENT}

The identification of certain company names in this paper does not imply endorsement by NIST.

\section{REFERENCES}

[1] J. S. Hoye and G. Stell, "Kerr effect. II. Contributions from hyperpolarizability and anisotropic polarizability," J. Chem. Phys., vol. 88, pp. 5798-5804, May 1988.

[2] D. Tang, A. H. Rose, G. W. Day, and S. M. Etzel, "Annealing of linear birefringence in single mode fiber coils: Application to optical fiber current sensors," J. Lightwave Technol., vol. 9, pp. 1031-1037, Aug. 1991.

[3] K. Stalsberg, W. Au. L. Strandjord, R. Goettsche, T. El-Wailly, G. Sanders, J. Blake, J. Demcko, and W. Chilton, "Fiber optic current sensor and multi-application data acquisition and analysis system," in Proc. Fault and Disturbance Analysis Conf., Atlanta, GA, May 1998.

[4] G. W. Day, M. N. Deeter, and A. H. Rose, "Faraday effect sensors: A review of recent progress," in Proc. SPIE, 1992, vol. PM07, pp. 11-26.

[5] T. Bosselmann, "Magneto-and electrooptic transformers meet expectations of power industry," in Proc. 12th OFS Conf., OSA Tech. Dig. Series, Oct. 1997, vol. 16, pp. 111-114.

[6] 3M Specialty Optical Fibers, "3M fiber optic current sensor module," Product Information Application Note, 1996.

[7] G. W. Day, K. B. Rochford, and A. H. Rose, "Fundamentals and problems of fiber current sensors," in Proc. OFS-11, Sapporo, Japan, May 1996, pp. 124-129.

[8] G. W. Day and A. H. Rose, "Faraday effect sensors: The state of the art," in Proc. SPIE, 1988, vol. 985, pp. 138-150.

[9] S. Donati, V. Annovazzi-Lodi, and T. Tambosso, "Magneto-optic fiber sensors for electrical industry: Analysis of performances," Inst. Elec. Eng. Proc. J., vol. 135, pp. 372-382, Oct. 1988.

[10] A. J. Rogers, "Optical methods of measurement of voltage and current on power systems," Opt. Laser Technol., vol. 9, pp. 273-283, 1977.

[11] M. Takahashi, H. Noda, K. Terai, S. Ikuta, Y. Mizutani, T. Yokota, T. Kaminishi, and T. Tamagawa, "Optical current transformer for gas insulated switchgear using silica optical fiber," IEEE Trans. Power Delivery, vol. 12, pp. 1422-1427, Oct. 1997.

[12] K. Bohnert, H. Brandle, and G. Frosio, "Field test of interferometric optical fiber high-voltage and current sensors," 10th OFS Conf. Proc. SPIE, Oct. 1994, vol. 2360, pp. 16-19.

[13] S. Kobayashi, A. Horide, I. Takagi, M. Higaki, G. Takahashi, E. Mori, and T. Yamagiwa, "Development and field test evaluation of optical current and voltage transformers for gas insulated switchgear," IEEE Trans. Power Delivery, vol. 7, pp. 815-821, Apr. 1992.

[14] E. Kakamura, K. Uchida, M. Koshiishi, T. Mitsui, S. Miyamoto, K. Nakamura, K. Itaka, T. Hara, and T. Yoda, "Development of fault section detecting system for gas insulated transmission lines," IEEE Trans. Power Delivery, vol. PWRD-1, pp. 19-26, Jan. 1986.

[15] Y. Kuroda, Y. Abe, H. Kuwahara, and K. Yoshinga, "Field test of fiberoptic voltage and current sensors applied to gas insulated substation," in Proc. SPIE, 1985, vol. 586, pp. 30-37.

[16] A. J. Rogers, "Optical-fiber current measurement," Int. J. Optoelectron., vol. 3, pp. 391-407, 1988 
[17] R. E. Hebner Jr., R. A. Malewski, and E. C. Cassidy, "Optical methods of electrical measurement at high voltage levels," in Proc. IEEE, Nov. 1977, vol. 65, pp. 1524-1548.

[18] G. R. Allen, "Kerr voltage sensor," in Proc. SPIE, 1986, vol. 648, pp. 213-227.

[19] M. Paillette, "Temperature-dependent behavior of the Kerr constant in the vitreous state," J. of Non-Crystalline Solids, vol. 91, pp. 253-258, 1987.

[20] A. Simon and R. Ulrich, "Evolution of polarization along a single-mode fiber," Appl. Phys. Lett., vol. 31, pp. 517-520, Oct. 1977.

[21] M. C. Farries and A. J. Rogers, "Temperature dependence of the Kerr effect in a silica optical fiber," Electron. Lett., vol. 19, pp. 890-891, Oct. 1983.

[22] H. Kirkham and S. Weikel, "Optical current transducers for power systems: A review," IEEE Trans. Power Delivery, vol. 9, pp. 1778-1788, Oct. 1994.

[23] T. Yoshino, Y. Ohno, and K. Kurosawa, "Design and application of fiber-optic electric and magnetic field sensors for high voltage electric system," in Proc., OFS-2, Stuttgart, Germany, Sept. 1984, pp. 55-58.

[24] N. Rajkumar, V. J. Kumar, and P. Sankaran, "Fiber sensor for the simultaneous measurement of current and voltage in a high-voltage system," Appl. Opt., vol. 32, pp. 1225-1228, Mar. 1993.

[25] R. T. de Carvalho and J. Blake, "Simultaneous measurement of electric and magnetic fields using a sagnac interferometer," in Proc. SPIE 10th OFS Conf., Oct. 1994, vol. 2360, pp. 411-414.

[26] Z. Wang, Y. Liao, S. Lai, and H. Zhao, "Fiber sensor for simultaneous measurement of current and voltage by single Lo-Bi fiber," in Proc. SPIE, 1996, vol. 2895, pp. 26-32.

[27] S. Huard, Polarization of Light. New York: Wiley, 1997.

[28] P. Menke and T. Bosselmann, "Temperature compensation in magnetoopic AC current sensors using an intelligent AC-DC signal evaluation," J. Lightwave Technol., vol. 13, pp. 1362-1370, July 1995.

[29] E. A. Ulmer, "A high-accuracy optical current transducer for electric power systems," IEEE Trans. Power Delivery, vol. 5, pp. 892-898, Apr. 1990.

[30] N. F. Borrelli, "Electric field induced birefringence in glasses," Phys. Chem. Glasses, vol. 12, pp. 93-96, Aug. 1971.

[31] ABB Power T\&D Company, CGIT/IPB Division, product literature, 1994.

[32] A. H. Rose, S. M. Etzel, and C. M. Wang, "Verdet constant dispersion in annealed optical fiber current sensors," J. Lightwave Technol., vol. 15, pp. 803-807, May 1997.

[33] Z. B. Ren, Ph. Robert, and P.-A. Paratte, "Temperature dependence of bend- and twist-induced birefringence in a low-birefringence fiber," Opt. Lett., vol. 13, pp. 62-64, Jan. 1988.

[34] W. J. Tropf, M. E. Thomas, and T. J. Harris, "Properties of crystals and glasses," Handbook of Optics, Vol. II, Devices, Measurements, and Properties, M. Bass, Ed. New York: McGraw-Hill, 1995, Table 19, ch. 33 , p. 33.35 .
[35] P. A. Williams, A. H. Rose, G. W. Day, T. E. Miller, and M. N. Deeter, "Temperature dependence of the Verdet constant in several diamagnetic glasses," Appl. Opt., vol. 30, pp. 1176-1178, Apr. 1991.

[36] D. A. Flavin, R. McBride, and J. D. C. Jones, "Absolute measurement of wavelengths from a multiplexed in-fiber Bragg grating array by shortscan interferometry," in Proc. 12th OFS Conf., OSA Tech. Dig. Series, Oct. 1997, vol. 16, pp. 24-27.

[37] J. Blake, P. Tantaswadi, and R. T. de Carvalho, "In-line Sagnac interferometer current sensor," IEEE Trans. Power Delivery, vol. 11, pp. 116-121, Jan. 1996

[38] S. X. Short, A. A. Tselikov, J. U. de Arruda, and J. N. Blake, "Imperfect quarter-waveplate compensation in Sagnac interferometer-type current sensors," J. Lightwave Technol., vol. 16, pp. 1212-1219, July 1998.

[39] A. H. Rose, Z. B. Ren, and G. W. Day, "Twisting and annealing optical fiber for current sensors," J. Lightwave Technol., vol. 14, pp. 2492-2498, Nov. 1996.

[40] G. W. Day, "Birefringence measurements in single mode optical fiber," in Proc. SPIE, 1982, vol. 425, pp. 72-79.

Allen H. Rose received the B.S. degree in physics at Abilene Christian University, Abilene, TX, in 1981 and the M.S. and Ph.D. degrees in physics at the University of Arkansas, Fayetteville, in 1983 and 1986.

In 1986, he joined the U.S. Army's Ballistic Research Laboratory, Aberdeen Proving Ground, MD, as an NRC Postdoctoral Fellow. In 1987, he joined the National Institute of Standards and Technology (NIST), then NBS, Boulder, $\mathrm{CO}$, to work on the development of optical fiber sensors, related optical measurements, and standards.

Dr. Rose has received an R\&D 100 Award and the NIST Bronze Medal for his research. He is a member of the Optical Society of America (OSA).

S. M. Etzel received the B.G.S. degree from the University of Iowa, Iowa City, IA, in 1987.

In 1978, she joined the National Institute of Standards and Technology (NIST), Boulder, CO, and has worked on polarimetry, laser power, and energy measurements as well as optical fiber characterization and research and development of optical sensors.

Kent B. Rochford (S'89-M'91), for a biography, see p. 836 of the May 1999 issue of this JOURNAL. 